\title{
Consumo de drogas en adolescentes con conductas infractoras: análisis de variables psicosociales implicadas
}

\section{Drug abuse in adolescent offenders: analysis of the psychosocial variables involved}

\author{
Lourdes Contreras MartíneZ; VIRGinIa Molina \\ Banqueri; Mª Carmen Cano Lozano
}

\author{
Departamento de Psicología, Universidad de Jaén.
Enviar correspondencia a:
Lourdes Contreras Martínez.
Universidad de Jaén. Campus Las Lagunillas. Edificio C5.
Despacho 017. 23071 JAÉN. \\ e-mail: Imcontre@ujaen.es
}

\section{Resumen}

Las investigaciones muestran que droga y delincuencia aparecen frecuentemente asociados, siendo una práctica habitual entre los menores infractores el consumo de distintos tipos de sustancias. A su vez, existen ciertas caracteristicas psicosociales que están relacionadas tanto con el consumo de drogas como con la conducta delictiva y/o antisocial. Nos planteamos por tanto en este estudio profundizar sobre la relación entre el consumo de drogas en menores infractores y ciertas variables del ámbito psicosocial de este colectivo. Para conseguir este objetivo se ha analizado el total de expedientes de menores infractores archivados en el Servicio de Justicia Juvenil de la provincia de Jaén, desde 2001 hasta 2010. De los expedientes consultados se han analizado variables demográficas como el género y la edad, asi como variables de tipo psicosocial como son el consumo de drogas, conducta violenta, grupo de iguales, el autocontrol, la tolerancia a la frustración y el estilo atribucional. Los resultados muestran que la mayoría de los menores infractores consumen algún tipo de sustancia, así como la existencia de diferencias en el patrón de consumo en función del género. También se observa que existe relación entre el consumo de sustancias y pertenecer a un grupo de iguales desviado, la conducta violenta y las variables psicológicas mencionadas, asi como diferencias en estas variables psicológicas en función del tipo de droga consumida.

Palabras clave: conducta delictiva, menores infractores, consumo de drogas, variables psicosociales.
Key words: criminal behavior, young offenders, drug abuse, psychosocial variables. 
$L^{3}$ a conducta delictiva juvenil ha sido ampliamente investigada desde el ámbito científico (Andrews y Bonta, 2006; Lösel y Bender, 2003; Simoes, Matos y BatistaFoguet, 2008). Existe un acusado interés por determinar cuáles son los factores y circunstancias que propician que un adolescente comience a mostrar conductas infractoras. Esta extensa investigación ha resultado en múltiples propuestas para explicar este tipo de comportamientos, formulándose diversas teorias desde diferentes perspectivas y enfoques científicos. Parece claro que no existe una única explicación para este fenómeno, de forma que son múltiples los factores vinculados a la conducta delictiva, siendo ésta en la mayoría de las ocasiones el resultado de interacciones dinámicas entre las diversas variables.

Una de las variables que en este sentido ha recibido más atención es el consumo de drogas en cuanto a su relación y/o influencia reciproca sobre este tipo de comportamientos. Droga y conducta antisocial y delictiva aparecen frecuentemente asociadas en diferentes investigaciones (Mulvey, Schubert y Chassin, 2010; Muñoz, Graña, Peña y Andreu, 2002; Simoes et al., 2008), encontrándose un patrón consistente de consumo de diferentes sustancias en los menores infractores y con conductas antisociales (Crespo, Perles y San Martín, 2006; Llorens, Palmer y Perelló del Río, 2005; Olivan, 2002; San Juan, Ocáriz y Germán, 2008). Según Loeber (1988) se produce un incremento en paralelo en el consumo de drogas y conducta antisocial, siendo este consumo más probable si la conducta antisocial iniciada durante la infancia persiste durante la adolescencia (Kandel, 1982). Cuando la conducta antisocial aparece a edades tempranas, y continúa en el comienzo de la adolescencia, puede ser considerada como un potente predictor de abuso de determinadas sustancias (Loeber, 1988). En otros estudios, los resultados muestran una relación a la inversa entre estas dos variables, puesto que se ha encontrado que la edad de inicio del consumo de sustancias es una variable predictiva de futuros consumos y actos violentos, de forma que los jóvenes que muestran conductas violentas comienzan a consumir drogas a edades más tempranas (Rivero, Marín e Infante, 2002). En cuanto a la influencia del género a la hora de consumir drogas, los estudios realizados con población adolescente general, indican que existen diferencias en cuanto al patrón de consumo de sustancias entre chicos y chicas. En este sentido, se ha observado que los chicos consumen en mayor proporción que las chicas todas las drogas ilegales, aunque en el consumo de drogas legales como el tabaco aparece un mayor número de consumidoras (Calvete y Estévez, 2009; Llorens et al., 2005; Observatorio Español sobre Drogas, 2009).

Así mismo, otra variable ampliamente investigada por su vinculación con la conducta delictiva durante la adolescencia tiene que ver con la relación con el grupo de iguales, que actúan como principales agentes de influencia durante esta etapa de la vida de la persona, ejerciendo como modelos tanto para la transmisión de valores positivos fundamentales (tolerancia, respeto, conductas prosociales, etc.), como para los valores negativos (no asunción de normas, desprecio por el respeto hacia los demás, ausencia de responsabilidad, etc.). De esta manera, el contacto y exposición a modelos de conducta antisocial se configura como uno de los principales factores de influencia para la comisión de actos antisociales y delictivos (Llinares y Benedito, 2007; Muñoz, 2004; Sobral, Romero, Luengo y Marzoa, 2000). A su vez, también se ha analizado la vinculación entre el consumo de drogas y el grupo de iguales, afirmándose en este sentido que la relación con grupos de iguales desviados y que consumen drogas facilita la adopción y mantenimiento de una actitud permisiva hacia el consumo de sustancias (Moral, Ovejero y Pastor, 2004), incrementando esto a su vez la aceptación y participación en conductas de riesgo (Wright y Fitzpatrick, 2004), incluyendo conductas delictivas de mayor gravedad (Kinlock, Battjes y Gordon, 2004). Por tanto, la relación con grupos de iguales desviados juega un papel central tanto en el consumo de drogas como en el desarrollo de conductas delictivas (Fishbein y Pérez, 2000).

Por otro lado, existen diferentes investigaciones que evidencian que algunas características psicológicas están relacionadas tanto con la conducta delictiva como con el consumo de drogas. Así, parece existir cierta relación entre un bajo autocontrol y la conducta delictiva, e incluso con la persistencia de esta conducta infractora, tal y como se muestra en recientes investigaciones (Contreras, Molina y Cano, 2011; Monahan, Steinberg, Cauffman y Mulvey, 2009; Mulvey et al., 2010). Los menores con conductas antisociales y/o delictivas presentan una combinación de alta impulsividad y bajo autocontrol, que resulta en una dificultad para demorar la gratificación de sus necesidades (López y López, 2003). El estilo atribucional es otra variable que ha sido vinculada a ciertos comportamientos inadaptados, de forma que los menores con este tipo de conductas tienden a atribuir sus actos a causas externas y a no asumir la responsabilidad sobre los mismos (Peterson y Leigh, 1990), de forma que el locus de control externo correlaciona con la conducta antisocial (Fariña, Seijo, Arce y Novo, 2002; Vázquez, Fariña y Arce, 2005). También se han relacionado otras variables, como por ejemplo el razonamiento moral (Espinosa, Clemente y Vidal, 2002) y ciertos aspectos de la conducta antisocial y delictiva. Además, como se ha mencionado anteriormente, otros estudios han analizado el papel de algunas de estas características psicológicas como son la impulsividad (Fantín, 2006), el autocontrol, el estilo atribucional y la asertividad (López-Torrecilla, Martín, De la Fuente y Godoy, 2000) como predictores del consumo de sustancias. Concretamente parece que son factores de riesgo, entre otros, poseer un locus de control externo (Alonso y Del Barrio, 1996), baja tolerancia a la frustración (Calvete y Estévez, 2009; González, GarciaSeñorán y González, 1996) y déficits en autocontrol (Espada, Méndez, Griffin y Botvin, 2003). Incluso se ha observado que existen algunas características de personalidad que influyen de forma diferencial en el tipo de sustancia consumida (Llorens et al., 2005).

Finalmente, algunos autores han analizado conjuntamente estas variables, encontrando relación entre consumo de sustancias y características psicológicas como la alta impulsividad y búsqueda de sensaciones y, a su vez, 
con la conducta antisocial de los adolescentes (Horovath y Zuckerman, 1993). Otros autores, profundizando aún más sobre esta cuestión, han observado que, concretamente, los problemas de conducta externalizantes son el factor de riesgo más importante para el consumo de drogas ilegales en menores infractores, y que esta relación está mediada por el consumo previo de otras drogas legales como son el tabaco y el alcohol (Helstrom, Bryan, Hutchison, Riggs y Blechman, 2004).

Existen pocos estudios que analicen conjuntamente el consumo de drogas y ciertas características psicológicas en menores infractores. Es necesario, por tanto, profundizar sobre este grave problema social que es el consumo de drogas en menores que además suelen cometer actos antisociales y delictivos, así como su relación con ciertas características que forman parte del entorno más inmediato de los mismos, ya que puede aportar información esencial a la hora de diseñar líneas de intervención con este colectivo. En consecuencia, nos planteamos como objetivo analizar el consumo de drogas en menores infractores y cuáles son las variables que están implicadas en este patrón de consumo. Concretamente, en primer lugar, se pretende analizar la prevalencia de consumo de drogas entre menores infractores y si existen diferencias en función del género y la edad. También nos interesa comprobar si el consumo de drogas en menores infractores está relacionado con algunas variables de tipo psicosocial, como son el grupo de iguales, la conducta violenta, locus de control, autocontrol y tolerancia a la frustración. Finalmente, nos planteamos analizar cuáles de estas variables están implicadas en la comisión de un delito violento frente un delito no violento. De acuerdo con las investigaciones previas realizadas sobre el tema, esperamos encontrar una mayor proporción de menores infractores que consumen drogas frente a los que no consumen y un mayor consumo en varones frente a las mujeres. También esperamos encontrar un mayor porcentaje de menores infractores que consumen drogas y que muestran conductas violentas, déficits en autocontrol, en tolerancia a la frustración y un locus de control externo, frente a los menores infractores que no son consumidores.

\section{Método}

\section{Muestra}

Para la realización de este estudio se analizaron el total de expedientes de Reforma de Menores (1046 menores) finalizados y archivados en el Servicio de Justicia Juvenil, Delegación Provincial de Justicia y Administración Pública de Jaén, desde la entrada en vigor de la L.0. 5/2000 hasta 2010. Del total de expedientes analizados se excluyeron 392 por ser resueltos extrajudicialmente y no incluir suficiente información. La muestra final fue de 654 menores, siendo 568 hombres y 86 mujeres, con una media de edad de 15,87 años y una desviación típica de 1,16.

\section{Diseño y procedimiento}

El presente trabajo es un estudio de tipo descriptivo mediante la consulta y análisis de documentos, de acuerdo con la clasificación propuesta por Montero y León (2005). La información consultada de los expedientes se recogió en una plantilla informática elaborada para tal fin. Del contenido del expediente se consultaron los informes de los técnicos de Reforma Juvenil sobre el inicio, ejecución y finalización de la medida judicial. De estos documentos se extrajo la información relativa a las variables que se presentan a continuación. Las categorias de cada variable se corresponden con las que aparecen en los informes de los técnicos:

- Variables demográficas: género y edad.

- Variables psicosociales: consumo de drogas (síno), tipo de drogas (alcohol/tabaco/cannabis/cocaina/policonsumo) (se considera policonsumo el consumo de tres o más sustancias, legales y/o ilegales), grupo de iguales (normalizado/ desviado), conducta violenta (sí/no), tolerancia a la frustración (normal/déficit), autocontrol (normal/déficit) y locus de control (interno/externo).

- Tipo de delito (violento/no violento).

Una vez finalizada la consulta y análisis de los expedientes de reforma, se procedió a un segundo análisis por un segundo evaluador. En este segundo análisis se revisó el diez por ciento de la totalidad de los expedientes, elegidos al azar. Finalizada la segunda revisión, se procedió a la comparación de los datos de ambos evaluadores para establecer el porcentaje de acuerdo entre los mismos. Se eliminaron aquellas variables en las que no existía el máximo acuerdo entre los dos evaluadores.

Para el análisis de datos se utilizó la versión 17.0 del programa estadístico "Statistical Package for Social Sciences (SPSS 17.0). En primer lugar se realizaron los análisis descriptivos que incluyen la frecuencia y el porcentaje de todas las variables, analizando si las distribuciones de frecuencias eran las esperadas a través del estadístico Chi-cuadrado. A su vez, se analizó la asociación entre las variables consumo de drogas/tipo de drogas y cada una de las siguientes variables: género, edad, grupo de iguales, conducta violenta, tolerancia a la frustración, locus de control y autocontrol, utilizando para ello el método de análisis de contingencias a través del índice de asociación $\operatorname{Phi}(\varphi)$. Finalmente se utilizó la técnica de regresión logística para una variable dependiente no métrica con objeto de identificar qué variables de las estudiadas predicen mejor tanto el consumo de drogas como la comisión de un delito violento. Se utilizó como variables dependientes o criterio el consumo de drogas y el tipo de delito y como variables independientes o predictoras el grupo de iguales, la conducta violenta, el locus de control, autocontrol y tolerancia a la frustración, utilizando el estadístico $R^{2}$ de Nagelkerke (Ato y López, 1996) y un nivel de significación de 0,05. 


\section{Resultados}

\section{Consumo de drogas y tipo de drogas}

La mayoría de los menores consumen drogas (58\%), encontrándose diferencias significativas en la distribución de frecuencia de consumidores y no consumidores $\chi^{2}(1, N=609)=398,9$, siendo lo más frecuente el policonsumo (32,2\%), seguido del consumo conjunto de tabaco y alcohol (23,9\%) (Véase tabla 1).

Tabla 1. Frecuencia y porcentaje de consumo de drogas y tipo de drogas

\begin{tabular}{lcc}
\hline \multicolumn{1}{c}{ Consumo de drogas } & Frecuencia & Porcentaje \\
\hline $\mathrm{Si}$ & 350 & 58 \\
$\mathrm{No}$ & 259 & 42 \\
\hline Total & 609 & 100 \\
\hline Tipo de drogas & $\mathrm{F}$ & $\mathrm{P}$ \\
\hline Alcohol & 14 & 4 \\
Tabaco & 72 & 20,5 \\
Cannabis & 21 & 6 \\
Tabaco y alcohol & 84 & 23,9 \\
Tabaco y cannabis & 20 & 5,7 \\
Alcohol y cannabis & 8 & 2,3 \\
Cannabis y cocaina & 4 & 1,1 \\
Policonsumo & 123 & 32,2 \\
No especificada & 14 & 4 \\
Disolvente & 1 & 0,3 \\
\hline Total & 351 & 100 \\
\hline
\end{tabular}

\section{Consumo de drogas, género y edad}

No se han encontrado diferencias estadísticamente significativas en la frecuencia del consumo de drogas en función del género $\mathcal{X}^{2}(1, N=609)=2,22$, pero sí en el tipo de droga $\mathcal{X}^{2}(9, N=351)=25,8 ; \varphi=0,27$. Mientras que en los hombres lo más habitual es el policonsumo (35\%), en el caso de las mujeres la sustancia que se consume con mayor frecuencia es el tabaco (45\%) (Véase tabla 2).

Por otra parte, se observan diferencias estadísticamente significativas en la distribución de frecuencias del consumo de drogas en función de la edad $\chi^{2}(3, N=609)=$ 9,$62 ; \varphi=0,126$, pero no en el tipo de sustancia consumida en función de la edad $\mathcal{X}^{2}(27, N=351)=18,2$. Los grupos de menores en los que se observa un mayor consumo son los de 16 años $(60,2 \%)$ y el de 17 años (64,1\%). Así mismo, en todos los grupos de edad lo más frecuente es el policonsumo, seguido del consumo de tabaco con alcohol y de sólo tabaco (Véase tabla 2).
Tabla 2. Porcentaje de consumo/ tipo de drogas, género y edad

\begin{tabular}{|c|c|c|c|c|c|c|}
\hline \multirow[b]{2}{*}{$\begin{array}{l}\text { Consumo de } \\
\text { drogas }\end{array}$} & \multicolumn{2}{|c|}{ Género } & \multicolumn{4}{|c|}{ Edad } \\
\hline & Hombres & Mujeres & 14 años & 15 años & 16 años & 17 años \\
\hline $\mathrm{Si}$ & 58,7 & 50 & 47,9 & 51,9 & 60,2 & 64,1 \\
\hline No & 41,3 & 50 & 52,1 & 48,1 & 39,8 & 35,9 \\
\hline Tipo de drogas & Hombres & Mujeres & 14 años & 15 años & 16 años & 17 años \\
\hline Alcohol & 3,6 & 7,1 & 6,7 & 2,4 & 2,1 & 5,5 \\
\hline Tabaco & 17,2 & 45,2 & 20 & 23,2 & 20,6 & 18,9 \\
\hline Cannabis & 6,5 & 2,4 & 6,7 & 6,1 & 5,2 & 6,3 \\
\hline $\begin{array}{l}\text { Tabaco y } \\
\text { alcohol }\end{array}$ & 24,9 & 16,7 & 24,4 & 24,4 & 23,7 & 23,6 \\
\hline $\begin{array}{l}\text { Tabaco y } \\
\text { cannabis }\end{array}$ & 5,8 & 4,8 & 1 & 4,9 & 9,3 & 5,5 \\
\hline $\begin{array}{l}\text { Alcohol y } \\
\text { cannabis }\end{array}$ & 1,9 & 4,8 & 2,2 & 2,4 & 3,1 & 1,6 \\
\hline $\begin{array}{l}\text { Cannabis y } \\
\text { cocaina }\end{array}$ & 1 & 2,4 & 2,2 & 0 & 0 & 2,4 \\
\hline Policonsumo & 35 & 11,9 & 35 & 31,7 & 33 & 30,7 \\
\hline No especificada & 3,9 & 4,8 & 2,2 & 4,9 & 2,1 & 5,5 \\
\hline Disolvente & 0,3 & 0 & 0 & 0 & 1 & 0 \\
\hline
\end{tabular}

\section{Consumo de drogas, grupo de iguales y conducta violenta}

Los resultados muestran que la amplia mayoria de los sujetos que consumen drogas tiene un grupo de iguales desviado $(75,8 \%)$, frente a un grupo de iguales normalizado $(24,2 \%)$. Con respecto a la asociación entre el consumo de drogas y el grupo de iguales, el análisis de contingencias indica que estas variables están asociadas $\chi^{2}(1, N=568)=48,9 ; \varphi=0,27$, siendo esta asociación estadisticamente significativa.

Encontramos que la gran mayoría de los/as menores que muestran conductas violentas son consumidores de alguna sustancia (75,3\%), mientras que en el grupo de menores que no son violentos habitualmente, sólo la mitad de los menores son consumidores (50,3\%), observándose una asociación estadísticamente significativa entre estas dos variables $\chi^{2}(1, N=589)=34,4 ; \varphi=0,23$.

\section{Consumo de drogas y tolerancia a la frustración, autocontrol y locus de control}

La mayoria de los sujetos que consumen algún tipo de sustancia tienen baja tolerancia a la frustración (71,5\%). También se observa que estas dos variables está asociadas $\chi^{2}(3, N=550)=42,2 ; \varphi=0,29$, siendo esta asociación estadísticamente significativa (Véase tabla 3 ).

A su vez, la mayoría de los menores consumidores/as tienen un autocontrol deficitario (67,6\%). También se observa que estas dos variables están asociadas de manera estadisticamente significativa $\chi^{2}(1, N=566)=34,9 ; \varphi=0,24$ (Véase tabla 3). 
Además, la mayoría de los menores con un locus de control externo son consumidores de alguna sustancia $(67,2 \%)$, encontrándose estas variables significativamente asociadas $\chi^{2}(1, N=566)=14,5 ; \varphi=0,16$ (Véase tabla 3).

Tabla 3. Porcentaje de consumo de drogas y tolerancia a la frustración, autocontrol y locus de control

\begin{tabular}{l|cccccc}
\hline \multirow{2}{*}{$\begin{array}{l}\text { Consumo de } \\
\text { drogas }\end{array}$} & \multicolumn{2}{|c}{ Tolerancia a la frustración } & \multicolumn{2}{c}{ Autocontrol * } & \multicolumn{2}{c}{ Locus de control * } \\
\cline { 2 - 7 } & Normal & Déficit & Normal & Déficit & Interno & Externo \\
\hline $\mathrm{Si}$ & 28,5 & 71,5 & 32,4 & 67,6 & 32,8 & 67,2 \\
\hline No & 57,6 & 42,4 & 57,3 & 42,7 & 51,3 & 48,7 \\
\hline${ }^{*} \mathrm{P}<0,05$ & & & & & &
\end{tabular}

\section{Tipo de droga consumida y tolerancia a la frustración, autocontrol y locus de control}

Se encuentran asociaciones estadísticamente significativas entre el tipo de droga consumida y la tolerancia a la frustración $\chi^{2}(18, N=320)=42,8 ; \varphi=0,34$, el autocontrol $\chi^{2}(9, N=328)=37,5 ; \varphi=0,32$ y el locus de control $\chi^{2}(9$, $N=332$ ) =46,007; $\varphi=0,35$ (Véase tabla 2). La mayoría de los menores que consumen cannabis o son consumidores de tres o más sustancias tienen déficits en todas las variables. Además, mientras que el consumo de sustancias como el tabaco no parece vinculado a déficits en ninguna variable, cuando los menores lo consumen junto con el cannabis muestran déficits tanto en tolerancia a la frustración (75\%), autocontrol (75\%) y un locus de control externo (70\%).

Finalmente, los análisis realizados mediante la técnica de regresión logística en cuanto al consumo de drogas nos indican que el modelo resulta estadísticamente significativo $\left(R_{\text {Nagelkerke }}^{2}=0,16, \mathrm{p}<0,001\right)$, siendo las variables que mejor predicen este consumo el grupo de iguales desviado $(p=0,002)$ y la conducta violenta habitual $(p=0,03)$. Con respecto al tipo de delito cometido (violento/no violento), los resultados de los análisis realizados muestran que el modelo es estadisticamente significativo $\left(R_{\text {Nagelkerke }}^{2}=0,08, \mathrm{p}<0,001\right)$, siendo las variables que mejor predicen el tipo de delito el grupo de iguales desviado $(p=0,002)$, un autocontrol deficitario $(p=0,004)$ y la conducta violenta habitual $(0,02)$.

\section{Discusión}

Los datos del presente estudio confirman la relación existente entre droga y conducta antisocial y delictiva, tal y como aparece reflejada en diferentes investigaciones (Mulvey et al., 2010; Muñoz et al., 2002; San Juan et al., 2009). Se observa la presencia de un mayor porcentaje de menores infractores que consumen algún tipo de sustancia frente a aquellos que no son consumidores. En cuanto al tipo de sustancia consumida, destaca el hecho de que lo más habitual en esta población sea el policonsumo, si bien también es frecuente el consumo exclusivo de tabaco y/o el alcohol. Estos datos están en línea con los ofrecidos por el Observatorio Español sobre Drogas en su informe de 2009 sobre consumo en la población general de adolescentes, en el que se indica que lo más habitual es que se consuman varias drogas, siendo el alcohol, tabaco y cannabis las más frecuentes. Con respecto a las diferencias entre hombres y mujeres, existe un patrón de consumo diferencial en cuanto al tipo de sustancia. En este sentido, en el grupo de hombres lo más habitual es el policonsumo, mientras que en el grupo de mujeres la sustancia consumida con mayor frecuencia es el tabaco. En otros estudios llevados a cabo con población adolescente general se obtienen resultados muy similares (Calvete y Estévez, 2009; Llorens et al., 2005; Observatorio Español sobre Drogas, 2009). Los datos de nuestra investigación también se asemejan en parte a los obtenidos por Martínez, Alonso y Montañés (2010), ya que en su estudio los chicos presentan mayores tasas de consumo de cannabis en comparación con las chicas, pero también consumen más cantidad de tabaco, a diferencia de nuestros resultados. Por tanto, podemos decir que el patrón de consumo de menores infractores se asemeja bastante al de la población general adolescente. En cualquier caso, los resultados indican que este consumo reviste una mayor gravedad en los varones, puesto que son los que consumen una mayor variedad de sustancias, frente a las mujeres que en su mayoría suelen consumir sólo tabaco.

En cuanto a la edad de los menores, la mayor frecuencia de consumo aparece en los grupos de 16 y 17 años, lo que es congruente con los datos de otras investigaciones que señalan que el consumo de sustancias se incrementa con la edad (Llorens et al., 2005; Observatorio Español sobre Dro-

Tabla 4. Porcentaje de tipo de droga consumida y variables psicológicas

\begin{tabular}{|c|c|c|c|c|c|c|c|}
\hline \multirow{2}{*}{$\begin{array}{c}\text { Variable psicológica } \\
\text { Tolerancia a la frustración }{ }^{*}\end{array}$} & \multicolumn{7}{|c|}{ Tipo de droga } \\
\hline & Alcohol & Tabaco & Cannabis & Tab.+Alc. & Tab.+Can & Can.+Coc. & Policon. \\
\hline Normal & 50 & 45,5 & 22,2 & 43,1 & 25 & 25 & 11,3 \\
\hline Déficit & 50 & 54,5 & 67,8 & 56,9 & 75 & 75 & 88,7 \\
\hline \multicolumn{8}{|l|}{ Autocontrol* } \\
\hline Normal & 50 & 49,3 & 15,8 & 48 & 30 & 25 & 15 \\
\hline Déficit & 50 & 50,7 & 84,2 & 52 & 70 & 75 & 85 \\
\hline \multicolumn{8}{|l|}{ Locus de control* } \\
\hline Interno & 46,2 & 72,9 & 27,8 & 63,3 & 30 & 50 & 32,1 \\
\hline Externo & 53,8 & 27,1 & 72,2 & 36,7 & 70 & 50 & 67,9 \\
\hline
\end{tabular}

${ }^{*} p<0,05$

$\mathrm{Tab}+\mathrm{alc}=$ tabaco + alcohol $/$ Policon $=$ politoxicomania $/$ Can.+Coc.=cannabis+cocaina $/ \mathrm{Tab} .+\mathrm{Can} . .=$ tabaco+cannabis 
gas, 2009). Sin embargo, en este trabajo la edad no parece ser una variable determinante a la hora de consumir diferentes tipos de sustancias, puesto que estos grupos presentan un patrón de consumo similar en este sentido, siendo lo más habitual en todos los menores el policonsumo independientemente de la edad del consumidor.

Por otra parte, se observa que la gran mayoría de los menores infractores que consumen algún tipo de sustancia poseen un grupo de iguales desviado, siendo esta variable una de las que mejor predicen el consumo de drogas. Esto confirma los resultados obtenidos en estudios previos en cuanto a la relación entre esta característica y conducta antisocial y/o delictiva (Llinares y Benedito, 2007; Muñoz, 2004; San Juan et al., 2009; Sobral et al., 2000), pudiendo actuar el grupo de iguales como reforzador de este tipo de conductas. Por tanto, droga, delincuencia y grupo de iguales desviado parecen estar de alguna manera vinculados, de forma que se confirma el hecho de que la relación con grupos de iguales desviados juega un papel central tanto en el consumo de drogas como en el desarrollo de conductas delictivas (Fishbein y Pérez, 2000). También se ha encontrado cierta asociación entre el consumo de drogas y la conducta violenta, siendo la conducta violenta habitual una de las variables que mejor predicen el consumo de sustancias en estos menores.

Tradicionalmente se ha encontrado que existen determinadas características psicológicas relacionadas tanto con la conducta delictiva (Contreras et al., 2011; Monahan et al., 2009; Mulvey et al., 2010) como con el consumo de drogas (Fantín, 2006; López-Torrecillas et al., 2000). En el presente trabajo los datos muestran que efectivamente existe esa relación, ya que la mayoría de los menores que consumen drogas tienen déficits tanto en el nivel de tolerancia a la frustración y autocontrol, así como un locus de control externo, datos que son congruentes con la literatura previa sobre el tema (Alonso y Del Barrio, 1996; Calvete y Estévez, 2009; Espada et al., 2003; Fariña et al., 2002; González et al., 1996; Vázquez et al., 2005). No obstante, nuestros resultados se asemejan en parte a los obtenidos por López-Torrecilla et al. (2000), que encontraron relación entre el consumo de drogas y el autocontrol, pero no con el estilo atribucional. Por tanto, existe alguna relación entre el consumo de sustancias y determinadas características psicológicas, y a su vez, con la conducta antisocial y/o delictiva, tal y como señalan Muñoz et al. (2002). También nos interesó analizar si existía alguna relación entre estas características psicológicas y el consumo de determinadas sustancias. Encontramos que la mayoria de los menores que consumen cannabis o son consumidores de tres o más sustancias tienen déficits en todas las variables. $\mathrm{El}$ consumo de drogas legales como son el tabaco y el alcohol no parecen estar vinculados a déficits en estas características. Sin embargo, cuando el consumo de tabaco se combina con el cannabis, la mayoría de los menores muestran déficits tanto en tolerancia a la frustración, autocontrol y en el estilo atribucional. A su vez, cuando el consumo de cannabis se combina con el de cocaína, se observan déficits tanto en la tolerancia a la frustración como en el autocontrol, pero no así en el estilo atribucional. Estos datos van en la línea de los aportados por Llorens et al. (2005) sobre la influencia diferencial de algunas características de personalidad en función del tipo de sustancia consumida.

A modo de conclusión, los resultados obtenidos aportan datos interesantes para la intervención con menores que han cometido algún tipo de delito o con menores en riesgo. Concretamente, el hecho que sean los chicos con edades comprendidas entre los 16 y 17 años los que consumen una mayor cantidad y/o variedad de sustancias puede ser tenido en cuenta a la hora de diseñar programas de prevención y/o de intervención en el consumo de drogas con esta población.

A su vez, los programas de intervención con menores infractores probablemente deberán incluir como uno de los objetivos fundamentales programas de deshabituación de consumo de drogas, puesto que la mayoría de los menores son consumidores, especialmente de cannabis. Además, los resultados del análisis conjunto del consumo de drogas/tipo de droga consumida, la conducta delictiva y características psicológicas, nos sugieren que dichas variables están vinculadas. De forma que, aunque la intervención es necesaria en todos los menores infractores en general, resulta prioritaria la intervención sobre las variables analizadas en aquellos menores infractores que consumen drogas, puesto que presentan mayores déficits que aquellos que no consumen.

Así mismo, es necesario mencionar algunas limitaciones de nuestra investigación. En primer lugar, no podemos asegurar la unanimidad de criterios de los técnicos de los Equipos Psicosociales en las valoraciones realizadas sobre las variables psicosociales incluidas, que han constituido nuestra única fuente de información al trabajar mediante la revisión de expedientes. No obstante, estas valoraciones son el producto del acuerdo entre al menos dos de estos técnicos y están basados en la administración de pruebas y en la categorización social. Por otra parte, en cuanto a la generalización de los resultados obtenidos, aunque estos datos se refieren a un área geográfica concreta, el gran volumen de expedientes analizados hace posible extraer conclusiones algo más generalizables, ya que se asemejan bastante a los obtenidos en otros estudios similares.

Finalmente, sería interesante en futuros estudios incluir un grupo de menores que no han cometido delitos para examinar el impacto diferencial de las variables psicosociales en la delincuencia juvenil. A su vez, se debería especificar el tipo de relación existente entre el consumo de drogas y las variables estudiadas, es decir, si el consumo de drogas es la causa o la consecuencia de poseer ciertas características psicosociales.

\section{Conflicto de intereses}

Los autores del artículo manifiestan no tener ningún conflicto de intereses. 


\section{Referencias}

Alonso, C. y Del Barrio, V. (1996). Efectividad de tres intervenciones para la prevención del consumo de alcohol en la escuela. Análisis y modificación de conducta, 24,679-701.

Andrews, D. A. y Bonta, J. (2006). The psychology of criminal conduct (4th ed.). Cincinnati, $\mathrm{OH}$ : Anderson Publishing Co.

Ato, M. y López, J. J. (1996). Análisis estadístico para datos categóricos. Madrid: Sintesis Psicología.

Calvete, E. y Estévez, A. (2009). Consumo de drogas en adolescentes: el papel del estrés, la impulsividad y los esquemas relacionados con la falta de limites. Adicciones, 21, 49-56.

Contreras, L., Molina, V. y Cano, M.C. (2011). In search of psychosocial variables linked to the recidivism in young offenders. The European Journal of Psychology Applied to Legal Context, 3, 77-88.

Crespo, M., Perles, F. y San Martín, J. (2006, noviembre). Análisis psicosocial de los menores infractores en un centro de reforma. Comunicación presentada en el / Congreso Europeo sobre Programas de Cumplimiento de Medidas Judiciales para Menores, Sevilla.

Espada, J. Méndez, J., Griffin, K. y Botvin, G. (2003). Adolescencia: consumo de alcohol y otras drogas. Papeles del Psicólogo, 84, 9-17.

Espinosa, P., Clemente, M. y Vidal, M.A. (2002). Conducta antisocial y desarrollo moral en el menor. Psicothema, 14, 26-36.

Fantín, M. B. (2006). Perfil de personalidad y consumo de drogas en adolescentes escolarizados. Adicciones, 18, 285-292.

Fariña, F., Seijo, D., Arce, R. y Novo, M. (2002). Psicología jurídica de la familia: Intervención en casos de separación y divorcio. Barcelona: Cedecs.

Fishbein, D. H. y Pérez, D. M. (2000). A regional study of risk factors for drug abuse and delinquency: sex and racial differences. Journal of Child and Family Studies, 9, 461-479. doi:10.1023/A:1009470825972.

González, F., Garcia-Señorán, M.M. y González. S. G. (1996). Consumo de drogas en la adolescencia. Psicothema, 8, 257-267.

Helstrom, A., Bryan, A., Hutchison, K.E., Riggs, P.D. y Blechman, E. A. (2004). Tobacco and alcohol use as an explanation for the association between externalizing behavior and illicit drug use among delinquent adolescents. Prevention Science, 5, 267-277. doi:10.1023/B:PREV.0000045360.23290.8f

Horvath, P. y Zucherman, M. (1993). Sensation seeking, risk appraisal, and risky behavior. Personality and individual differences, 14, 41-52. doi:10.1016/0191-8869(93)90173-Z

Kandel, D.B. (1982). Epidemiological and psychosocial perspectives on adolescent drug use. Journal of American Academic Clinical Psychiatry, 21, 328-347. doi:10.1016/S0002-7138(09)60936-5

Kinlock, T.W., Battjes, R.J. y Gordon, M. S. (2004). Factors associated with criminal severity among adolescents entering substance abuse treatment. Journal of Drug Issues, 34, 293-318.

Llinares, L. y Benedito, M.A. (2007). El grupo de iguales como contexto de inadaptación. Acciones e investigaciones sociales, 24, 65-99.

Llorens, N., Palmer, A. y Perelló del Río, M. J. (2005). Caracteristicas de personalidad en adolescentes como predictores de la conducta de consumo de sustancias psicoactivas. Trastornos adictivos, 7, 90-96.

Loeber, R. (1988). Natural histories of conduct problems, delinquency and associated substance use: evidence for developmental progressions. En B.B. Lahey y A. E. Kazdin (Eds.), Advances in clinical child psychology (pp.73-124). Nueva York: Plenum.
López, C., y López, J. (2003). Rasgos de personalidad y conducta antisocial y delictiva. Psicopatología Clinica, Legal y Forense, 3, 5-19.

López-Torrecilla, F., Martín, I., De la Fuente, I. y Godoy, J. F. (2000). Estilo atribucional, autocontrol y asertividad como predictores de la severidad en el consumo de drogas. Psicothema, 12, 331-334.

Lösel, F. y Bender, D. (2003). Protective factors and resilience. En D. P. Farrington y J. W. Coid (Eds.), Early prevention of antisocial behaviour (pp. 130-204). Cambridge, MA: Cambridge University Press.

Martínez, M., Alonso, C. y Montañés, J. (2010). Consumo de tabaco, alcohol y cannabis en adolescentes Castellano-Manchegos [resumen]. Revista española de drogodependencias, 1, 78-91.

Monahan, K.C., Steinberg, L., Cauffman, E. y Mulvey, E.P. (2009). Trajectories of antisocial behavior and psychosocial maturity from adolescence to young adulthood. Developmental Psychology, 45, 1654-1668. doi:10.1037/a0015862

Montero, I. y León, O. G. (2005). Sistema de clasificación del método en los informes de investigación en Psicología. International Journal of Clinical and Health Psychology, 5, 115-127.

Moral, M.V., Ovejero, A. y Pastor, J. (2004). Modelado familiar y del grupo de iguales sobre la experimentación juvenil con sustancias psicoactivas. Boletín de Psicología, 81, 33-68.

Mulvey, E. P., Steinberg, L., Piquero, A.R., Besana, M., Fagan, J., Schubert, C. y Cauffman, E. (2010). Trajectories of desistance and continuity in antisocial behavior following court adjudication among serious adolescent offenders. Development and Psychopathology, 22, 453-475. doi:10.1017/S0954579410000179

Mulvey, E.P., Schubert, C. A. y Chassin, L. (2010). Substance use and delinquent behavior among serious adolescent offenders. Office of Juvenile Justice and Delinquency Prevention. Recuperado el 19 de enero de 2011 de: http://www.ncjrs.gov/pdffiles1/ojjdp/232790.pdf

Muñoz, J.J. (2004). Factores de riesgo y protección de la conducta antisocial en adolescentes. Revista de Psiquiatría de la Facultad de Medicina de Barna, 31, 21-37.

Muñoz, M.J., Graña, J.L., Peña, M.E. y Andreu, J.M. (2002). Influencia de la conducta antisocial en el consumo de drogas ilegales en población adolescente. Adicciones, 14, 313-320.

Olivan, G. (2002). Estado de salud de jóvenes varones delincuentes. Atención Primaria, 29, 421-424.

Observatorio Español sobre Drogas de la Delegación de Gobierno para el Plan Nacional sobre Drogas (2009). Informe sobre la situación y tendencias de los problemas de drogas en España. Madrid: Ministerio de Sanidad y Política social. Recuperado el 20 de febrero de http://www.pnsd.msc.es/Categoria2/observa/pdf/oed2009.pdf.

Peterson, G.W. y Leigh, G.K. (1990). The family and social competence in adolescence. En T.P. Gullota, G.R. Adams y R. Montemayor (Eds.), Developing social competence in adolescence. Advances in adolescent development (Vol. 3, pp. 97-139). Thounsand Oaks, CA: Sage.

Rivero, M., Marín, M. e Infante, E. (2002). Tipo y trayectoria de consumo de drogas, edad de inicio y comportamiento violento en jóvenes. Revista Electrónica Interuniversitaria de Formación del Profesorado, 5, 1-7. Recuperado el 2 de junio de 2011 de: http:// www.dialnet.es

San Juan, D., Ocáriz, E. y Germán, I. (2009). Menores infractores y consumo de drogas: Perfil psicosocial y delictivo. Revista Criminalidad, 51, 147-162. 
Simoes, C., Matos, M. y Batista-Foguet (2008). Juvenile Delinquency: Analysis of risk and protective factors using quantitative and qualitative methods. Cognition, Brain, Behavior: An Interdisciplinary Journal, 12, 389-408.

Sobral, J., Romero, E., Luengo, A. y Marzoa, J. (2000). Personalidad y conducta antisocial: amplificadores individuales de los efectos contextuales. Psicothema, 12, 661-670.
Vázquez, M. J., Fariña, F. y Arce, R. (2003). Principales factores de riesgo y protección del comportamiento agresivo y antisocial. En F. Fariña y R. Arce (Eds.), Avances en torno al comportamiento antisocial, evaluación y tratamiento (pp.104-139). Madrid: Ministerio de Trabajo y Asuntos Sociales.

Wright, D.R., y Fitzpatrick, K.M. (2004). Psychosocial correlates of substance use behaviors among African American youth. Adolescence, 39, 653-667. 